\title{
VALORES Y ACTITUDES SOCIALMENTE RESPONSABLES EN UNIVERSITARIOS CHILENOS
}

\author{
Gracia Navarro, ${ }^{1}$ Paula Boero, ${ }^{2}$ Gladys Jiménez,${ }^{3}$ Liliana \\ Tapia, ${ }^{4}$ Reinier Hollander, ${ }^{5}$ Arturo Escobar, ${ }^{6}$ Margarita \\ Baeza, ${ }^{7}$ Álvaro Espina
}

RESUMEN

Como aporte a la comprensión del comportamiento socialmente responsable en estudiantes universitarios, el estudio pretende identificar sus valores y actitudes hacia la responsabilidad social; determinar diferencias según sexo, promoción y área de pertenencia de la carrera e identificar la relación entre valores, actitudes hacia esta y el comportamiento socialmente responsable. Se encuestó a 2.876 estudiantes de seis universidades chilenas con CAVSR y CACSR (Proyecto MECESUP UCO0303, 2004-2008). Los resultados muestran una relación positiva muy significativa entre valores, actitudes y comportamiento socialmente responsable; compromiso con valores y actitudes vinculados a las vivencias próximas a los universitarios y menor compromiso con aquellos vinculados al bienestar social, la ciudadanía, democracia y participación. Se observan, además, diferencias significativas según sexo, promoción y área de pertenencia de la carrera. Como conclusión, se observa la necesidad de impulsar prácticas educativas que activen valores menos preponderantes y promuevan actitudes positivas hacia ellos a fin de lograr un ejercicio pleno de la responsabilidad social.

Palabras clave: valores, actitudes, responsabilidad social, universitarios

\section{SOCIALLY-RESPONSIBLE VALUES AND ATTITUDES IN CHILEAN UNIVERSITY STUDENTS}

\begin{abstract}
As a contribution to comprehension of the socially responsible behaviour in college students, this study tries to identify their values and attitudes towards social responsibility; to determine differences according to gender, promotion and career areas and to identify the relationship among values, attitudes towards social responsibility and a socially responsible behaviour. It surveyed 2.876 students from six Chilean universities by CAVSR and CACSR (Project MECESUP UCO0303, 2004-2008). Results show a very positive and significant relation among values, attitudes and socially responsible behaviour, commitment with values and attitudes that are closer to college student experiences and less commitment with those linked to social wellbeing, citizenship, democracy and participation. There are significant differences according to gender, promotion and career areas. As a conclusion, there is a need to stimulate educational practices that activate less preponderant values and promote positive attitudes towards them in order to achieve a social responsibility full exercise.
\end{abstract}

Keywords: values, attitude, social responsibility, college students

1 Universidad de Concepción. Concepción, Chile. Contacto: gnavarro@udec.cl

2-7Universidad de La Frontera

3 Pontificia Universidad Católica de Valparaíso

4 Universidad de Valparaíso

5 Universidad del Bío-Bío

6 Universidad Austral de Chile 


\section{VALORES Y ACTITUDES SOCIALMENTE RESPONSABLES EN UNIVERSITARIOS CHILENOS}

\section{Introducción}

Al igual que lo visto en Navarro, Boero, Jiménez, et al. (2010), la responsabilidad social (RS) es conceptualizada como "la orientación de las actividades individuales y colectivas en un sentido que permita a todos igualdad de oportunidades para desarrollar sus capacidades, eliminando y apoyando la eliminación de obstáculos estructurales de carácter económico y social, así como los culturales y políticos que afectan o impiden ese desarrollo" (Urzúa, 2001, pp. 2-3). Desde la psicología individual, Berman (1997) entiende a la RS como el compromiso personal con los demás e incluye tres dimensiones necesarias para comprometerse con otros, esto es: (a) entender que cada individuo pertenece a una red de relaciones sociales más amplia cuya influencia es decisiva en la construcción de la propia imagen de sí mismo; (b) mantener relaciones interpersonales basadas en consideraciones éticas de justicia y de preocupación por los otros; (c) así como actuar con integridad, de manera consistente con los propios valores, en otras palabras es la inversión personal en el bienestar propio, de otros y del planeta. En lo efectivo, Navarro (2006, p. 86) define como conductas socialmente responsables aquellas "que pueden darse en diferentes ámbitos, que tienen a la base una intención orientada hacia el bienestar de todos y que se ejercen con una determinada frecuencia".

En el contexto del sistema educativo, la RS ha sido promovida por la UNESCO a partir de la Declaración de la Conferencia Mundial de Educación Superior de 1998 y ratificada en 2009. Específicamente en la educación superior, la RS exige a las instituciones la comprensión de materias que presentan múltiples dimensiones, considerando las categorías sociales, económicas, científicas y culturales, y las habilidades para responder a ellas. En general, el concepto de RS señala que la educación superior debería incrementar su mirada 
interdisciplinaria y promover el pensamiento crítico y una ciudadanía activa para contribuir al logro del desarrollo sustentable, la paz, el bienestar y los derechos humanos, incluyendo la equidad de género. También, señala que la educación superior no solo debería proveer competencias sólidas al mundo presente y futuro, sino que debe contribuir a la educación de ciudadanos éticos, comprometidos con la construcción de la paz, la defensa de los derechos humanos y los valores de la democracia.

De manera similar, el Proyecto Tuning Latinoamérica (2007) ha considerado a la "responsabilidad social y el compromiso ciudadano" dentro de las 27 competencias genéricas prioritarias para la educación superior. En el caso específico de la universidad, forma parte de su misión crear conocimiento y formar los científicos, humanistas y profesionales orientados a satisfacer las necesidades de desarrollo de un país (Proyecto Universidad Construye País, 2006). Así visto, temas como la pobreza, la desintegración social, el desarrollo del capital social y el desarrollo sustentable deberían estar en el centro de sus preocupaciones (Torres y Trápaga, 2010). No obstante lo anterior, es posible observar algo distinto a esto: que priman tendencias orientadas por el poder, el utilitarismo y el éxito personal en detrimento de los valores como el respeto, la fraternidad, la honestidad, la solidaridad y la responsabilidad para con la sociedad (Equipo Coordinador Universidad Construye País, 2002).

Una investigación realizada en 5.515 estudiantes de seis universidades chilenas (Navarro, Boero, Jiménez et al., 2010) mostró en estos una baja autoatribución de comportamientos socialmente responsables, destacando la convivencia social, por su alta frecuencia de realización y orientación hacia el bienestar de todos, entre aquellos comportamientos incluidos en una de las diez categorías medidas. Esta se refiere a los comportamientos de una vida en comunidad acorde con las normas y necesidades individuales y grupales. La misma investigación mostró diferencias significativas para el comportamiento socialmente responsable entre hombres y mujeres, a favor de estas; diferencias significativas según la promoción, con mayor frecuencia de conductas socialmente responsables e intención de bien común, en alumnos de cuarto año en comparación a los de primero y, diferencias 
significativas según el área de pertenencia de la carrera (ciencias sociales-humanidades; químico-biológica y físico-matemática); los estudiantes de las carreras del área de ciencias sociales-humanidades junto al área químico-biológica, mostraron una mayor autoatribución de comportamiento socialmente responsable e intenciones más colectivistas respecto de los estudiantes del área físico-matemática.

La reflexión sobre estos resultados llevó a preguntarse cuáles serían las motivaciones subyacentes a este comportamiento y en este sentido, a investigar los valores y las actitudes que los influyen, ya que si bien las conductas son mediadas por una variedad de factores, se sabe que los valores juegan un papel motivacional importante en al menos cuatro aspectos: a) en la búsqueda de consistencia entre sus creencias (valores) y sus conductas (Rokeach, 1973); b) influyendo en el planeamiento de las acciones (Schwartz, 2007); c) influyendo en la atención, la percepción y, por tanto, en la interpretación de situaciones (Schwartz, 2005); y también, d) ayudando a las personas a determinar lo que quieren (Schwartz, 2007). Si bien los valores se adquieren por medio de la socialización, es tarea personal jerarquizarlos, especialmente en situaciones de conflicto o dilema moral, demostrando en este proceso la competencia moral de cada persona (Martí, 2010). Del mismo modo, la relación entre conductas y actitudes ha sido ampliamente estudiada y documentada (McBroom y Reed, 1992; Morales, 1999) enfatizando las interacciones de los diferentes componentes de la actitud: cognitivo, afectivo y conativo conductual y sus relaciones con la conducta final que desarrolla el sujeto. A partir de lo anterior, surgen las siguientes preguntas de investigación: ¿cuáles son los valores y las actitudes asociados a la responsabilidad social en los universitarios chilenos? y ¿existe relación entre los valores, las actitudes y el comportamiento socialmente responsable?

Esta investigación tiene por objetivos: a) identificar los valores y las actitudes hacia la responsabilidad social en estudiantes universitarios chilenos; b) determinar si existen diferencias significativas en valores y actitudes hacia la responsabilidad social según sexo, promoción y área de pertenencia de la carrera y c) determinar si existe relación entre valores, actitudes hacia la responsabilidad social y comportamiento socialmente responsable. 


\section{Método}

\section{Participantes}

La muestra estuvo constituida por 2.876 estudiantes de primer y cuarto año pertenecientes a 56 carreras de las seis universidades chilenas, adscritas al proyecto MECESUP UCO0303. Ellos representan el 4,4\% del total de alumnos matriculados en esas instituciones de educación superior. De ellos 55,2\% son mujeres, 42,6\% de hombres y $2,2 \%$ no dio información al respecto. Los sujetos tienen una edad promedio de 20,1 años. El 15,4\% de los encuestados pertenece a las carreras clasificadas dentro del área físico-matemática; 43,5\% son de carreras del área químico-biológica y $41 \%$ al área de ciencias sociales y humanidades. El 79,2\% corresponde a estudiantes de primer año y $20,8 \%$ a cuarto año, muestreados con el fin de establecer comparaciones entre estudiantes principiantes y estudiantes en pleno proceso formativo.

Tabla n. ${ }^{\circ}$ 1: Distribución de la muestra por sexo y promoción

\begin{tabular}{|l|c|c|c|}
\hline Sexo & \multicolumn{3}{|c|}{ Promoción } \\
\hline & $1^{\text {er }}$ año & $4^{\circ}$ año & Total \\
\hline Hombres & 991 & 235 & 1226 \\
\hline Mujeres & 1241 & 347 & 1588 \\
\hline S/R & 46 & 16 & 62 \\
\hline Total & 2.278 & 598 & 2.876 \\
\hline
\end{tabular}

Tabla n. ${ }^{\circ}$ 2: Distribución de la muestra por sexo y área de pertenencia de la carrera

\begin{tabular}{|l|c|c|c|c|}
\hline Sexo & \multicolumn{4}{|c|}{ Área de pertenencia } \\
\hline & Fco-Mat. & Qco-Biol. & Csoc-Hum. & Total \\
\hline Hombres & 334 & 449 & 443 & 1.226 \\
\hline Mujeres & 105 & 770 & 713 & 1.588 \\
\hline S/R & 6 & 32 & 24 & 62 \\
\hline Total & 445 & 1.251 & 1.180 & 2.876 \\
\hline
\end{tabular}

\section{Variables e instrumentos}

Valores y actitudes hacia la responsabilidad social: se utilizó el cuestionario diseñado para medir actitudes y valores asociados a la responsabilidad social en estudiantes universitarios (CAVSR), que fuera elaborado para el proyecto MECESUP UCO0303 (2005). Está compuesto por dos escalas que miden 11 dimensiones valóricas. 
1) Escala de valores: considera 66 ítems, cada uno con una pequeña definición entre paréntesis y se pregunta: "como principio que guía mi vida, ieste valor es?". Las respuestas son de tipo likert de 9 opciones: desde -1 (opuesto a mis valores) a 0 (nada de importante) 4 (importante) hasta 7 (de suma importancia). La asimetría de la escala manifiesta la discriminación que la gente naturalmente hace cuando piensa acerca de la importancia de un valor (Bardi y Schwartz, 2003). A mayor puntaje, mayor importancia otorgada al ítem. Anexo A.

2) Escala de actitudes: consta de 33 ítems y se pregunta sobre el grado de acuerdo con la frase presentada como dispositivo. Las respuestas son de tipo likert de 5 opciones, desde 1 (totalmente en desacuerdo) a 3 (indiferente) hasta 5 (totalmente de acuerdo). A mayor puntaje, mayor grado de acuerdo con el ítem. Anexo B.

Ambas escalas tienen una hoja de respuesta independiente que incluye datos demográficos y celdillas correspondientes a las opciones de respuesta entregadas.

Las dimensiones valóricas fueron construidas teóricamente y se refieren a dignidad de la persona; libertad; ciudadanía, democracia y participación; sociabilidad y solidaridad para la convivencia; bien común y equidad social; desarrollo sostenible y medio ambiente; aceptación y aprecio de la diversidad; compromiso con la verdad; integridad; excelencia; interdependencia e interdisciplinariedad (Anexo C).

La confiabilidad medida a través del Coeficiente de Alpha de Cronbach es de 0,79 para la Escala de Actitudes, y de 0,95 para la Escala de Valores. La validez, para ambas escalas, fue verificada mediante el método interjueces.

Comportamiento socialmente responsable: se utilizó el cuestionario para evaluar comportamientos socialmente responsables en estudiantes universitarios (CACSR), que fuera elaborado en la Universidad de Concepción por Davidovich, Espina, Navarro et al. (2005). Está compuesto por dos escalas que miden: 
1) La frecuencia de comportamiento socialmente responsable (E1) en cada una de las siguientes categorías: responsabilidad académica; actividades de voluntariado; ayuda social; actividades religiosas; convivencia social; responsabilidad cívico-universitaria; autocuidado; desarrollo cultural; ecología y medio ambiente; respeto por los espacios compartidos.

2) La intención de comportamiento socialmente responsable (E2), que puede ser: beneficio para todos, beneficio individual, beneficio para los demás o no tiene claridad en la intención.

La confiabilidad medida por medio del Coeficiente de Alpha de Cronbach es de 0,82 para la E1, y de 0,61 para la E2. La validez fue verificada mediante el método interjueces.

\section{Procedimiento}

La aplicación se realizó en paralelo en las universidades de Concepción, del Bío-Bío, de La Frontera, Austral de Chile, de Valparaíso y Pontificia Católica de Valparaíso, durante el año 2005. El procedimiento fue estandarizado, mediante manuales tanto para los encuestadores como para el coordinador de aplicación y el coordinador general de evaluación, en cada una de las universidades participantes. La aplicación fue realizada en forma colectiva en sala de aula, donde los estudiantes fueron informados acerca del objetivo de la evaluación, su carácter voluntario y la confidencialidad en el tratamiento de los datos. La aplicación del CAVSR tuvo una duración aproximada de 60 minutos. Finalmente, los datos del conjunto de universidades fueron procesados de forma centralizada, utilizando el software estadístico SPSS (Statistical Package for the Social Sciences, versión 13).

\section{Análisis de datos}

Para el análisis de datos se obtuvieron las puntuaciones medias para ambas escalas, y luego se establecieron las diferencias entre los grupos de acuerdo a las variables sexo, área de pertenencia y promoción cursada por los estudiantes. Específicamente, se establecieron diferencias para sexo y promoción mediante una prueba $t$ para grupos 
independientes. Y para la variable área de pertenencia se realizó un Análisis de Varianza (ANOVA).

La prueba tevalúa si existe una diferencia significativa entre las medias de dos condiciones. En este caso se evaluó, para la variable sexo, la existencia de diferencias estadísticamente significativas entre el grupo de hombres y el grupo de mujeres, con relación a las escalas medidas. Y también para la variable promoción, se midió la existencia de diferencias significativas entre los estudiantes de primer año y los estudiantes de cuarto año, con respecto de las mismas escalas. En el caso del ANOVA, evalúa si existe una diferencia significativa entre alguna o todas las medias de los grupos testeados, comparándolas con la media global, cuando hay más de dos grupos que se comparan. En este caso, se evaluó la existencia de diferencias significativas entre los grupos de estudiantes pertenecientes a las tres áreas de pertenencia (químico-biológica, físico-matemática y de ciencias sociales y humanidades) con relación a las escalas medidas.

\section{Resultados}

1. Valores y las actitudes hacia la responsabilidad social

A continuación se exponen las puntuaciones medias para ambas escalas y luego se establecerán las diferencias de grupos de acuerdo a las variables sexo, área de pertenencia y promoción cursada por los estudiantes.

Los resultados de la Escala de Valores (tabla n. ${ }^{\circ}$ 3) muestra que los estudiantes evalúan los valores examinados bajo la premisa de "principios que guían la propia vida", de forma "muy importante" e "importante". Las dimensiones valóricas prioritarias en orden decreciente son: "libertad" $(M=6,89 ; D E=0,97)$, "dignidad de la persona" ( $M=6,81 ; D E=0,96)$ y "compromiso con la verdad" ( $M$ $=6,62 ; D E=1,02)$, hasta finalizar con "ciudadanía, democracia y participación" ( $M=5,40 ; D E=1,29)$ que, aunque se la considera importante, es la menos significativa de todas. 
Tabla n. ${ }^{\circ}$ 3: Puntuaciones promedio de la Escala de Valores en las 11 dimensiones valóricas del CAVSR en estudiantes universitarios

\begin{tabular}{|l|c|c|c|c|c|}
\hline Dimensiones valóricas & N & Mín. & Máx. & Media & DE \\
\hline Libertad & 2875 & 1,00 & 16,83 & 6,8998 & 0,97133 \\
\hline Dignidad de las personas & 2874 & 1,00 & 8,00 & 6,8167 & 0,96202 \\
\hline Compromiso con la verdad & 2873 & 1,00 & 17,67 & 6,6292 & 1,02250 \\
\hline Excelencia & 2874 & 1,00 & 12,17 & 6,5182 & 1,01333 \\
\hline Bien común y equidad & 2873 & 0,00 & 20,17 & 6,4896 & 1,17201 \\
\hline Solidaridad y sociabilidad & 2875 & 1,00 & 12,50 & 6,2973 & 1,12546 \\
\hline Desarrollo sostenible & 2874 & 1,00 & 11,67 & 6,2195 & 1,17310 \\
\hline Integridad & 2874 & 1,00 & 8,00 & 6,1096 & 1,12496 \\
\hline Interdependencia & 2874 & 1,00 & 17,50 & 5,8162 & 1,13795 \\
\hline Aceptación de la diversidad & 2874 & 0,00 & 18,17 & 5,8087 & 1,22321 \\
\hline Ciudadanía, democracia y participación & 2874 & 0,00 & 14,33 & 5,4059 & 1,29697 \\
\hline Escala de Valores & 2875 & 1,00 & 8,00 & 6,3028 & 0,86195 \\
\hline
\end{tabular}

Los resultados para la Escala de Actitudes (tabla n. ${ }^{\circ}$ 4), que mide el grado de acuerdo con una determinada dimensión, refleja que los universitarios reportan actitudes que van desde un grado de indiferencia como, por ejemplo, para la dimensión "dignidad de la persona" ( $M$ $=3,00 ; D E=0,95)$ o cercana a ella como en el caso de "ciudadanía, democracia y participación" ( $M=3,20 ; D E=0,74)$. Hasta reportar un grado mayor de acuerdo en las dimensiones "integridad de la persona" $(M=4,22 ; D E=0,70)$, "desarrollo sostenible" $(M=4,18 ; D E=0,72) y$ "bien común y equidad" ( $M=4,17 ; D E=0,73)$. No se observa ninguna dimensión con la que el grado de acuerdo o desacuerdo sea total.

Tabla n. ${ }^{\circ}$ 4: Puntuaciones promedio para la Escala de Actitudes en las 11 dimensiones valóricas del CAVSR en estudiantes universitarios

\begin{tabular}{|l|c|c|c|c|c|}
\hline Dimensiones valóricas & $\mathrm{N}$ & Mín. & Máx. & Media & DE \\
\hline Integridad & 2872 & 1,00 & 5,00 & 4,2241 & 0,70432 \\
\hline Desarrollo sostenible & 2872 & 1,00 & 5,00 & 4,1805 & 0,72847 \\
\hline Bien común y equidad & 2872 & 1,00 & 18,67 & 4,1744 & 0,73012 \\
\hline Interdependencia & 2871 & 1,00 & 5,00 & 4,0161 & 0,67377 \\
\hline Solidaridad y sociabilidad & 2870 & 1,00 & 5,00 & 3,9981 & 0,68330 \\
\hline Libertad & 2872 & 1,00 & 5,00 & 3,8332 & 0,71746 \\
\hline Excelencia & 2872 & 1,00 & 10,00 & 3,7233 & 0,67223 \\
\hline Aceptación de la diversidad & 2872 & 1,00 & 7,00 & 3,6556 & 0,78715 \\
\hline Compromiso con la verdad & 2872 & 1,00 & 20,67 & 3,3992 & 0,84711 \\
\hline Ciudadanía, democracia y participación & 2871 & 1,00 & 5,00 & 3,2003 & 0,74499 \\
\hline Dignidad de las personas & 2872 & 1,00 & 5,00 & 3,0011 & 0,95063 \\
\hline Escala de Actitudes & 2872 & 1,52 & 5,52 & 3,7641 & 0,40000 \\
\hline
\end{tabular}


2. Valores y actitudes hacia la responsabilidad social según sexo, promoción y área de pertenencia de la carrera

Respecto de los resultados por sexo, ambas escalas revelan que las mujeres expresan en general un mayor grado de acuerdo que los hombres con las dimensiones presentadas, así como también le otorgan mayor importancia a los valores examinados como principios rectores de la vida (tabla n. ${ }^{\circ} 5$ ). Ambas diferencias resultaron ser muy significativas para la Escala de Actitudes $(t=14,634 ; \mathrm{p}=0,000)$ y para la Escala de Valores ( $t=10,291 ; \mathrm{p}=0,000)$.

Tabla n. ${ }^{\circ}$ 5: Puntuaciones promedio de los universitarios en las escalas de valores y actitudes del CAVSR según sexo

\begin{tabular}{|l|c|c|c|}
\hline Sexo & N & Media & DE \\
\hline Escala de Valores Mujer & 1583 & 6,4448 & 0,80600 \\
\hline Hombre & 1220 & 6,1080 & 0,89873 \\
\hline Escala de Actitudes Mujer & 1582 & 3,8567 & 0,39980 \\
\hline Hombre & 1220 & 3,6412 & 0,38199 \\
\hline
\end{tabular}

Un examen al interior de la Escala de Valores, evidencia que las mujeres atribuyen una mayor importancia que los hombres a los valores "sociabilidad y solidaridad para la convivencia" $(t=15,599 ; \mathrm{p}=0,000)$, "aceptación a la diversidad" ( $t=11,846$; $\mathrm{p}=0,000)$, "interdependencia" ( $t=5,373 ; \mathrm{p}=0,000)$, "integridad" ( $t=$ 7,324; $\mathrm{p}=0,000)$, "excelencia" ( $t=4,479 ; \mathrm{p}=0,000)$, "dignidad de las personas" ( $t=10,981 ; \mathrm{p}=0,000)$, "bien común y equidad" $(t=8,706$; $\mathrm{p}=0.000)$, "desarrollo sostenible" $(t=5,678 ; \mathrm{p}=0,000)$ y "libertad" $(t=7,229 ; \mathrm{p}=0,000)$. Además, se agregan las diferencias a favor de las mujeres para las dimensiones "ciudadanía, democracia y participación" $(t=3,612 ; \mathrm{p}=0,000)$ y "compromiso con la verdad" $(t=8,741 ; \mathrm{p}=0,000)$.

La misma situación ocurrió en la Escala de Actitudes, en donde se reveló que las mujeres presentan diferencias muy significativas respecto de los hombres en las dimensiones "sociabilidad y solidaridad para la convivencia" ( $t=13,347 ; \mathrm{p}=0,000)$, "aceptación y aprecio de la diversidad" ( $t=13,389 ; \mathrm{p}=0,000)$, "interdependencia" ( $t=6,517$; $\mathrm{p}=0,004)$, "integridad" ( $t=6,699 ; \mathrm{p}=0,000)$, "excelencia" $(t=5,694$; $\mathrm{p}=0,000)$, "dignidad de las personas" ( $t=7,934 ; \mathrm{p}=0,000)$, "bien común y equidad" ( $t=7,716 ; \mathrm{p}=0,000)$, "desarrollo sostenible" $(t=3,211 ; \mathrm{p}=0,001)$ y "libertad" $(t=9,522 ; \mathrm{p}=0,000)$. 
Los resultados según área de pertenencia de los estudiantes muestran que, en ambas escalas, el área de ciencias sociales y humanidades, otorga mayor importancia y reporta mayor grado de acuerdo con las dimensiones presentadas con relación a las otras dos áreas (Escala de Valores $M=6,37 ; D E=0,84$ y Escala de Actitudes $M=3,79 ; D E=0,42$ ). Los estudiantes del área físico-matemática son los que exhiben las medias más bajas para las Escalas de Valores y Actitudes $(M=6,04 ; D E=0,87)$ y $(M=3,62 D E=0,37)$, respectivamente, diferencias que fueron estadísticamente muy significativas en ambas escalas $(\mathrm{F}=24,448 ; \mathrm{p}=0,000)$ y $(\mathrm{F}=37,075 ; \mathrm{p}=0,000)$.

Respecto de la promoción cursada por los estudiantes, los resultados para ambas escalas constatan diferencias entre los grupos de primer y cuarto año, sin embargo estas no fueron significativas.

3. Relación entre valores, actitudes hacia la responsabilidad social y comportamiento socialmente responsable

Finalmente, al correlacionar los datos del CAVSR se observó que existe una correlación positiva, muy significativa aunque baja entre sus escalas, $(\mathrm{r}=0,384 ; \mathrm{p}=0,000)$. Luego, al correlacionar las escalas del CAVSR con las escalas del cuestionario de comportamientos socialmente responsables (CACSR), los resultados mostraron correlaciones positivas, muy significativas pero también bajas, como se muestra en la tabla n. ${ }^{\circ} 6$. 
Tabla n. ${ }^{\circ}$ 6: Correlaciones entre las escalas de CAVSR y las escalas de CACSR

\begin{tabular}{|c|c|c|c|c|c|}
\hline & & $\begin{array}{c}\text { Escala l } \\
\text { Frecuencia de } \\
\text { comportamiento }\end{array}$ & $\begin{array}{c}\text { Escala } 2 \\
\text { Intención de } \\
\text { comportamiento }\end{array}$ & $\begin{array}{l}\text { Escala de } \\
\text { Actitudes }\end{array}$ & $\begin{array}{c}\text { Escala de } \\
\text { Valores }\end{array}$ \\
\hline $\begin{array}{l}\text { Escala } 1 \\
\text { Frecuencia de } \\
\text { comportamiento }\end{array}$ & $\begin{array}{l}\text { Correlación de } \\
\text { Pearson } \\
\text { Sig. (bilateral) } \\
\text { N }\end{array}$ & 1 & $\begin{array}{c}0,378^{* *} \\
0,000 \\
5515\end{array}$ & $\begin{array}{c}0,357^{* *} \\
\\
0,000 \\
1657 \\
\end{array}$ & $\begin{array}{c}0,326^{* *} \\
0,000 \\
1657\end{array}$ \\
\hline $\begin{array}{l}\text { Escala } 2 \\
\text { Intención de } \\
\text { comportamiento }\end{array}$ & $\begin{array}{l}\text { Correlación de } \\
\text { Pearson } \\
\text { Sig. (bilateral) } \\
\text { N }\end{array}$ & $\begin{array}{l}0,378 \\
0,000 \\
5515 \\
\end{array}$ & 5518 & $\begin{array}{c}0,287^{* *} \\
\\
0,000 \\
1653 \\
\end{array}$ & $\begin{array}{c}0,206^{* *} \\
0,000 \\
1651 \\
\end{array}$ \\
\hline $\begin{array}{l}\text { Escala de } \\
\text { Actitudes }\end{array}$ & \begin{tabular}{|l|} 
Correlación de \\
Pearson \\
Sig. (bilateral) \\
N \\
\end{tabular} & $\begin{array}{c}0,357^{* *} \\
0,000 \\
1657 \\
\end{array}$ & $\begin{array}{c}0,287^{* *} \\
0,000 \\
1653 \\
\end{array}$ & 2883 & $\begin{array}{c}0,384^{* *} \\
0,000 \\
2877 \\
\end{array}$ \\
\hline Escala de Valores & \begin{tabular}{|l} 
Correlación de \\
Pearson \\
Sig. (bilateral) \\
N
\end{tabular} & $\begin{array}{c}0,326^{* *} \\
0,000 \\
1655 \\
\end{array}$ & $\begin{array}{c}0,206^{* *} \\
0,000 \\
1651 \\
\end{array}$ & $\begin{array}{c}0,384^{* *} \\
0,000 \\
2877 \\
\end{array}$ & 2881 \\
\hline
\end{tabular}

** La correlación es significativa a nivel 0,01 (bilateral).

\section{Discusión}

Considerando que los objetivos de esta investigación fueron: a) identificar los valores y las actitudes hacia la responsabilidad social en estudiantes universitarios chilenos; b) determinar si existen diferencias significativas en valores y actitudes hacia la responsabilidad social según sexo, promoción y área de pertenencia de la carrera y c) determinar si existe relación entre valores, actitudes hacia la responsabilidad social y comportamiento socialmente responsable.

\section{Con relación a los valores y actitudes hacia la} responsabilidad social en los estudiantes de la muestra

La coincidencia entre 9 de 11 valores y actitudes hacia la responsabilidad social, es coherente con los estudios que señalan que los valores, así como influyen sobre el comportamiento, también influyen sobre las actitudes (Schwartz, 2005), sin embargo, como ya se ha documentado y también se percibe por medio de este artículo, estas relaciones tienen una complejidad que va más allá de la mera causalidad directa (McBroom y Reed, 1992; Schwartz, 2005, 2007). 
Con relación al grado de importancia otorgado a las dimensiones valóricas, pudiera obedecer a dos hipótesis: la primera, se vincula con la percepción que se tiene sobre ellas como aspectos más cercanos o más lejanos de la propia experiencia. La segunda, ligada a la primera explicación, puede relacionarse con el momento evolutivo de los encuestados. Ambas tesis pueden explicarse en función de la propuesta de Verplanken y Holland, (2002) citados por Schwartz (2005), quienes plantean que los valores necesitan ser activados para afectar el comportamiento. La probabilidad de que un valor sea activado va a depender, en parte, de su accesibilidad, es decir, de la facilidad con que son evocados. De forma que los valores más accesibles son más importantes para las personas. En este caso, se observa que las dimensiones valóricas consideradas "de suma importancia" son aquellas relacionadas al ámbito más próximo de los encuestados: "libertad", "dignidad de la persona", "compromiso con la verdad" y "excelencia". Todos valores vivenciados muy de cerca, algunos de ellos experimentados con especial intensidad durante este periodo como en el caso de la libertad, ya que, si se considera que un $79,2 \%$ son estudiantes de primer año de universidad y que la edad promedio es de 20,1 años, es, para muchos de ellos, la primera vez que tienen la oportunidad de decidir respecto de sus recursos, tiempos, objetivos y acciones, como los expresan los ítems que representan esta dimensión: libertad de acción (poder hacer lo que se quiere); libertad de pensamiento (pensar de manera independiente); libertad de expresión (poder expresar lo que se piensa); responsabilidad ante la vida (hacerse cargo de las consecuencias de los propios actos) y libertad de asociación (tener la facultad de elegir con quienes establecer relaciones). Asimismo, la "dignidad de las personas", esto es, la valoración de las personas, el autorrespeto y la consideración social son aspectos relevantes debido al nuevo estatus que han adquirido en este medio social. Del mismo modo, "compromiso con la verdad" y "excelencia" son aspectos solicitados a diario en sus actividades universitarias.

Los valores considerados "muy importantes", se relacionan con temas que incumben a la sociedad y que desde allí se promueven activamente: "bien común y equidad", "solidaridad y sociabilidad para la convivencia", "desarrollo sostenible y medio ambiente", e 
"integridad". Finalmente, los valores considerados “importantes" están vinculados con aspectos o concepciones que son un poco más distantes en su vida diaria como: la" interdependencia e interdisciplinaridad", "aceptación y aprecio de la diversidad" y finalmente, como se reportó "ciudadanía, democracia y participación".

En cuanto a aquellas dimensiones en que se observa una diferencia entre los valores y las actitudes, como en el caso de la "ciudadanía, democracia y participación", considerada muy importante, pero frente a la que se tiene una actitud casi neutra $(M=3,20 ; D E=$ $0,74)$, y "dignidad de la persona", cuya actitud es neutra $(M=3,00$; $D E=0,95)$, es relevante constatar que no se trata de un desacuerdo de los encuestados con esas dimensiones valóricas, sino más bien se trata de indiferencia. En este sentido, los resultados pueden ser abordados desde dos perspectivas complementarias: una sociológica, como una característica de los individuos posmodernos en que, como propone Bauman (2004), la indiferencia se deriva de un grado de tolerancia extrema, y también desde una perspectiva psicológica relativa a los componentes de la actitud. En el primer caso, como explica Bauman (2004), se trataría de una actitud que incluye la búsqueda de la buena vida, sin exigencias personales ni sociales y una actitud de tolerancia extrema que solo reflejaría una genuina indiferencia hacia la sociedad general, puesto que las personas suelen estar centradas en sí mismas, observándose, autovalorándose, luego estas dimensiones adquieren un carácter autorreferencial característico del individualismo.

En términos psicológicos, estos fenómenos pueden explicarse debido a que los componentes de la actitud: cognitivo, afectivo y conativo conductual, no siempre concuerdan entre sí frente a la evaluación del objeto de la actitud (Morales, 1999). Pudiendo predominar cualquiera de los tres componentes, por separado o en conjunto frente al objeto de actitud, en este caso frente a las dimensiones valóricas, o como se observará en el segundo caso en que existe una compensación interna para llegar a constituir una actitud neutra.

En el primer caso, "ciudadanía, democracia y participación" es interesante observar que en cuanto medida de valor, esta dimensión 
obtuvo la puntuación más baja en esa escala, es decir, en comparación a todas las otras dimensiones valóricas es la menos importante de ellas. Con relación a las actitudes, un examen al interior de los ítems revela que tanto el componente afectivo de la actitud "la posibilidad de estar inscrito en el registro electoral me llena de satisfacción" $(M=2,95 ; D E=1,31)$, como el componente conativo conductual de la actitud "evito asistir a asambleas estudiantiles cuando tengo la posibilidad de hacer algo más entretenido" $(M=2,77 ; D E=1,18)$ muestran una evaluación casi neutra y solo el componente cognitivo revela una evaluación levemente más positiva $(M=3,87 ; D E=0,85)$. Este resultado también coincide y de alguna manera explica los datos obtenidos por los mismos autores en la medición de comportamientos socialmente responsables (Navarro, Boero, Jiménez, et al. 2010), que reveló que la categoría que se relaciona teóricamente con la dimensión "ciudadanía, democracia y participación", la "responsabilidad cívico universitaria" referida a comportamientos vinculados al ejercicio de los derechos y deberes cívicos, se practique solo "a veces". Es decir, aunque la democracia, el ejercicio de la ciudadanía y la consiguiente participación son temas que vinculan activamente a los universitarios al mundo social; en primer lugar, son percibidos como un valor menos importante que otras dimensiones y, en segundo lugar, tanto los componentes afectivo y conativo conductual de la actitud son neutros debilitando el ejercicio de esta conducta y, en ese sentido, puede llegar a constituirse en una explicación de la tan criticada baja participación ciudadana y desinterés por la política en los jóvenes chilenos como lo demuestran los recientes estudios, entre ellos, los datos proporcionados por Rengifo (2008) y Jiménez y Sánchez (2008), quienes reportan que más del $48 \%$ de los jóvenes no participan de ninguna institución y solo 2,2\% lo hace en una organización política.

En el segundo caso, la dimensión "dignidad de la persona", en donde la actitud de indiferencia encontrada contrasta con la valoración de suma importancia otorgada por los estudiantes, puede ser explicada por compensación entre los ítems que constituyen los diferentes componentes de la actitud. El componente afectivo de la actitud representado por el ítem "me enorgullece reconocer que las personas tenemos distintos derechos de acuerdo con los méritos que hemos hecho para alcanzarlos" fue evaluado en desacuerdo $(M=2,35$; 
$D E=1,27)$, es decir, no se es más digno por tener más méritos lo que coincide con la valoración positiva. En cambio, el ítem que apunta al componente conductual de la actitud "demuestro respeto a las personas, solo si ellas me parecen dignas de ello" ( $M=3,40 ; D E=1,44)$ muestra un leve grado de acuerdo que refuerza la idea del carácter autorreferencial de la actitud como se expuso anteriormente y, por último, el aspecto cognitivo de la actitud "pienso que el crecimiento y el desarrollo personal constituyen más bien una opción que un derecho de las personas" $(M=3,24 ; D E=1,42)$ se mostró casi neutro. El resultado fue una puntuación promedio que reflejó una medida de tendencia central de clara indiferencia $(M=3,00 ; D E=0,95)$, mostrando, al igual que en el caso anterior, que todos los componentes de la actitud son relevantes y que de su combinación va a resultar la actitud que finalmente se expresará. En este caso, además, se confirma el peso de la perspectiva individual de la forma en que la plantea Bauman (2004), es decir, como una falsa tolerancia.

\section{Con relación a diferencias en valores y actitudes hacia la responsabilidad social según sexo, promoción y área de pertenencia de la carrera}

Las diferencias observadas respecto de la importancia otorgada a las dimensiones valóricas y el grado de acuerdo expresado con ellas, entre los estudiantes que estudian carreras pertenecientes a diferentes áreas (ciencias sociales y humanidades; químico-biológica y físicomatemática) coincide con los resultados obtenidos por Navarro, Boero, Jiménez, et al. (2010) en la medición de comportamiento socialmente responsable. Es posible explicar estas diferencias en función de que, en el caso de las dos primeras áreas, se desarrolla tempranamente un mayor contacto con las personas en términos de los requerimientos de la propia formación profesional, es decir, estudiantes de carreras como psicología, odontología y pedagogía, entre otras, tienen la oportunidad de interactuar con otros desde muy temprano en su formación profesional, lo que sin duda cumple un papel de activador de los valores y, por tanto, estos se tornan más visibles e importantes. Junto a ello, la necesidad de desarrollar conductas pro sociales y cooperativas, requeridas por la profesión, gravitan en una toma de perspectiva social y se fortalece, entonces, 
el proceso de desarrollo moral (Comunian y Gielen, 2006) y, por tanto, los valores y actitudes asociados a él. En cambio, en el caso de las carreras del área físico-matemática, este contacto solo comienza en una etapa muy avanzada de su formación, y si a eso se suma la mayor cantidad de varones que cursa estas carreras se agrega un elemento más que explica este contraste. Pues, como ya se describió en los resultados existen diferencias significativas entre hombres y mujeres.

Estas diferencias entre sexos, en lo que respecta a la responsabilidad social, han sido documentadas en investigaciones previas, a favor de las mujeres (Nadler, Romek y Shapira-Friedman, 1979; Bustamante y Navarro, 2007; Navarro, Boero, Jiménez et al., 2010). Del mismo modo, en la pesquisa actual es interesante constatar que tanto en la medición de los valores como de las actitudes, las mujeres obtienen diferencias significativas respecto de los hombres en casi todas las dimensiones, así como se observó también para los comportamientos socialmente responsables. Comenzando con la "sociabilidad y solidaridad para la convivencia"; la "aceptación y aprecio de la diversidad"; y la "interdependencia e interdisciplinariedad”, es decir, en aquellos valores y actitudes que se relacionan con la ética de cuidado, así como afirma la tesis de Gilligan (1992). Pues, si se observan las definiciones de esas dimensiones, ellas se refieren a la participación activa en la comunidad, al compromiso que supone la convivencia mutua, a la práctica de la solidaridad, al aprecio de lo diverso, a valorar a los otros y también a integrarlos. Así como también al establecimiento de relaciones entre los individuos y la sociedad, reconociendo la necesidad de esa mutualidad y la necesidad de diálogo permanente. Esto es, interactuar con los otros en función del cuidado del otro y de su bienestar. En palabras de Gilligan (1992), las mujeres se definirían a sí mismas con relación al mundo, describiéndose por medio de acciones que las ponen en contacto con los demás, elaborando nexos por medio de su capacidad de ayudar, es decir, su yo es delimitado por la conexión con otros. De manera que en sus acciones morales prevalecen las premisas sobre el cuidado, la inclusión y el respeto a las personas. 


\section{Acerca de la relación entre valores, actitudes hacia la responsabilidad social y comportamiento socialmente responsable}

Cabe destacar que todas las dimensiones valóricas medidas se relacionan con el comportamiento socialmente responsable de forma positiva y muy significativa, tanto en la importancia atribuida como "principio que guía mi vida" ( $\mathrm{r}=0,326 ; \mathrm{p}=, 000)$, como en el grado de acuerdo expresado respecto de estas dimensiones $(\mathrm{r}=0,357 ; \mathrm{p}=, 000)$, aunque, como se ha visto, lo hacen con una baja intensidad.

Estos resultados si bien no eran esperados por los investigadores, coinciden con los que otros autores han obtenido cuando relacionan dimensiones valóricas y conducta. Concretamente, Bardi y Schwartz (2003), en un estudio sobre la intensidad y estructura de las relaciones entre valores y conducta, mostraron que los valores se diferencian en sus correlaciones con diversos tipos de conducta. Estos hallazgos, según los autores, pueden ser interpretados como una señal de que la relación entre valores y conducta puede esconder presiones normativas y, también, que los comportamientos no son guiados por la prioridad de un solo valor, sino que ellos interactúan simultáneamente y, por tanto, hay que examinar cada caso de forma particular (Schwartz, 2005).

Si se considera esta última premisa, junto al antecedente de que los valores actuarían como activadores de la conducta, es posible explicar por qué la "convivencia social" calificó como única categoría de comportamiento considerada socialmente responsable en la medición previa con el CACSR. La "convivencia social" se refiere a los comportamientos relativos al ejercicio de una vida en comunidad acorde con las normas y necesidades individuales y grupales, a la tolerancia de los jóvenes a la diversidad, a su respeto hacia otros en la comunicación, en las normas sociales y, al mismo tiempo, de la percepción de que son capaces de expresar sus opiniones sobre temas diversos con libertad, es decir, junto con el respeto a otros existe la apreciación de que pueden ser respetados. Así, observamos una clara vinculación con las dimensiones valóricas de la "libertad" y "solidaridad y sociabilidad para la convivencia", ambas consideradas prioritarias y con un alto grado de acuerdo. 
Aun cuando los valores y las actitudes asociados a la responsabilidad social presentan correlaciones positivas, muy significativas pero bajas, coinciden en nueve de las once dimensiones medidas y aparecen vinculadas a las vivencias que los estudiantes universitarios experimentan de forma más próxima así como a algunos comportamientos socialmente responsables previamente medidos. Es importante llamar la atención sobre la menor prioridad otorgada a las dimensiones valóricas relativas a la vida en sociedad, a la verdadera tolerancia y a la interdependencia, pues ello puede ayudar a entender la estrecha y limitada percepción que la sociedad actual parece estar construyendo de sí misma, lo que, como evidencian Bauman (2004) y otros pensadores, puede responder a una ética basada en la autorreferencia individualista característica de estos tiempos posmodernos, que ya se había reportado en el estudio sobre comportamiento socialmente responsable (Navarro, Boero, Jiménez et al., 2010). Uno de los reflejos más notorios de esto es la baja participación en las instituciones y procesos cívicos que se observa en Chile en estas últimas décadas. Asimismo, es posible observar los resultados contrarios, cuando los estudiantes tempranamente entran en contacto con personas distintas a sus grupos mediante el ejercicio profesional, como se vio en el caso de las carreras de las áreas de ciencias sociales, humanidades y biológica, pues estas experiencias apoyan el desarrollo de valores, actitudes y conductas asociadas a la responsabilidad social. En este sentido, este estudio aporta elementos que ayudan al proceso de reflexión sobre el tipo de sociedad que se está construyendo, sus prioridades valóricas y las prácticas que se motivan a partir de ellas.

La baja correlación interescalas entre el CAVSR y CACSR constituye una limitación, pues ello impide explorar relaciones más complejas entre valores, actitudes y conductas, y sus interacciones recíprocas, y como consecuencia de ello, construir explicaciones más elaboradas acerca de esas interacciones. Por tanto, es un tema sobre el cual se tendrá que seguir profundizando tanto desde la perspectiva metodológica como empírica con nuevos estudios en universitarios. Finalmente, se desprende de estos resultados y su análisis, la necesidad de impulsar currículos y prácticas educativas específicas, como el aprendizaje y servicio, apoyadas en la experiencia y la reflexión 
conjunta, que activen también los valores y promuevan actitudes positivas frente a temas ligados a la protección del bienestar de las personas y del entorno, como el "bien común", la "equidad social", el "desarrollo sostenible" y la "ciudadanía, democracia y participación", con el fin de lograr un pleno ejercicio de la responsabilidad social.

\section{Referencias bibliográficas}

Bardi, A.; Schwartz, S. (2003). Values and behavior: Strength and structure of relations [versión electrónica]. Personality and Social Psychology Bulletin, 29, pp. 1207-1220.

Bauman, Z. (2004). Ética posmoderna. Buenos Aires: Siglo XXI Editores Argentina S.A.

Berman, S. (1997). Children's social consciousness and the development of social responsibility. New York: New York State University Press.

Bustamante, M.J.; Navarro, G. (2007). Autoatribución de comportamientos socialmente responsables de estudiantes de carreras del área de ciencias sociales. Revista Perspectivas, 18, pp. 45-63.

Comunian, A.; Gielen, U. (2006). Promotion of moral judgement maturity through stimulation of social role-taking and social reflection: an Italian intervention study [versión electrónica]. Journal of Moral Education, 35, 1, pp. 51-69.

Equipo Coordinador Universidad Construye País (2002). Marco Conceptual sobre la Responsabilidad Social Universitaria. Santiago, Chile: Autor.

Gilligan, C. (1992). Uma voz diferente: Psicologia da diferença entre homens e mulheres da infância a idade adulta. Rio de Janeiro: Rosa dos Tempos.

Jiménez, S.; Sánchez, R. (2008). La participación política de la juventud chilena. Entre las pautas clásicas y el desapego a sus instituciones [versión electrónica]. Revista observatorio de juventud 5, 19, pp. 18-31.

McBroom, W.; Reed, F. (1992). Toward a Reconceptualization of AttitudeBehavior Consistency [versión electrónica]. Social Psychology Quarterly, 55, 2, pp. 205-216.

Martí, M. (2010). Razonamiento moral y prosocialidad. Madrid: Editorial CCS.

Morales, J. F. (1999). Psicología Social. Madrid: McGraw-Hill.

Nadler, A.; Romek, E.; Shapira-Friedman, A. (1979). Giving in the kibbutz: Pro social behavior of city and kibbutz children as affected by social responsibility and social pressure. Journal cross cultural psychology, 10,1, pp. 57-72. 
Navarro, G.; Boero, P.; Jiménez, G.; Tapia, L.; Hollander, R.; Escobar, A.; Baeza, M.; Espina, A. (2010). Universitarios y Responsabilidad Social. Revista Calidad en la Educación, 33, pp. 101-121.

Navarro, G. (2006). Comportamiento socialmente responsable. En Responsabilidad social universitaria, una manera de ser universidad, teoría y práctica de la experiencia chilena. Santiago, Chile: Proyecto Universidad Construye País.

Navarro, G.; Jiménez, G.; Tapia, L. et al. (2003). Formación de profesionales con valores, actitudes y comportamiento que favorezcan el ejercicio de su responsabilidad social. Proyecto MECESUP UCO 0303.

Proyecto Universidad Construye País (2006). Responsabilidad social universitaria, una manera de ser universidad, teoría y práctica de la experiencia chilena. Santiago, Chile: Autor.

Proyecto MECESUP UCO0303 (2005). Cuestionario de actitudes y valores asociados a la responsabilidad social: Informe de construcción y validación. Manuscrito no publicado, Universidad de Concepción, Chile.

Rengifo, E. (2008). Juventud y Participación política en Chile. Del ciudadano al consumidor, un tránsito forzado, versión electrónica. Revista observatorio de juventud 5, 19, pp. 7-17.

Rokeach, M. (1973). The nature of human values. New York: Free Press.

Schwartz, S. (2005). Validade e aplicabilidade da Teoria de Valores. Em A. Tamayo y J. Porto (Orgs.) Valores e comportamentos nas organizações, pp. 56-95. Petrópolis: Editora Vozes.

Schwartz, S. (2007). Universalism values and the inclusiveness of our moral universe, [versión electrónica]. Journal of cross cultural psychology 38, 6, pp. 711-728.

Torres, M. y Trápaga, M. (2010). Responsabilidad social de la Universidad; retos y perspectiva. Barcelona: Editorial Paidós.

Tuning América Latina (2007). Reflexiones y perspectivas de la educación superior en América Latina. Consultado en http://tuning.unideusto. org/tuningal

UNESCO (1998). Declaración mundial sobre la educación superior en el siglo XXI: visión y acción. Consultado en http://unesdoc.unesco.org/ images/0011/001147/114723s.pdf\#114729

UNESCO (2009). Conferencia mundial de educación superior 2009: las nuevas dinámicas de la educación superior y de la investigación para el cambio social y el desarrollo. Consultado en http://www.unesco. org/education/WCHE2009/comunicado_es.pdf 
144 VALORES Y ACTITUDES SOCIALMENTE RESPONSABLES EN UNIVERSITARIOS CHILENOS - G. Navarro, P. Boero, G. Jiménez, L. Tapia, R. Hollander, A. Escobar, M. Baeza, Á. Espina

Urzúa, R. (2001, octubre). La responsabilidad social de las universidades: una guía para la discusión. Documento presentado en el taller de elaboración de estrategias para la expansión de la responsabilidad social en las universidades chilenas, Santiago de Chile, Chile.

Recibido: 11/02/2011

Aceptado: 03/05/2012 


\section{Anexo A: Ejemplos de ítems Escala Valores CAVSR}

\begin{tabular}{|c|c|}
\hline Dimensiones valóricas & Ejemplos de ítems \\
\hline \multirow{3}{*}{$\begin{array}{l}\text { Ciudadanía, } \\
\text { democracia y } \\
\text { participación. }\end{array}$} & Conciencia social (reflexionar sobre las problemáticas de la sociedad) \\
\hline & Participación (tomar parte en las decisiones de la comunidad) \\
\hline & Responsable cívicamente (cumplir los deberes y obligaciones personales) \\
\hline \multirow{3}{*}{$\begin{array}{l}\text { Dignidad de la } \\
\text { persona }\end{array}$} & Autorespeto (creer en el valor personal) \\
\hline & Valoración de las personas (apreciar el valor de todas las personas) \\
\hline & Consideración social (recibir el respeto y la aprobación de los demás) \\
\hline \multirow[t]{3}{*}{ Libertad } & Libertad de pensamiento (pensar de manera independiente) \\
\hline & Libertad de expresión u opinión (poder expresar lo que se piensa) \\
\hline & $\begin{array}{l}\text { Responsabilidad ante la vida (hacerse cargo de las consecuencias de los } \\
\text { propios actos) }\end{array}$ \\
\hline \multirow{3}{*}{$\begin{array}{l}\text { Solidaridad y } \\
\text { sociabilidad para la } \\
\text { convivencia }\end{array}$} & Colectivismo (búsqueda del beneficio mutuo) \\
\hline & Empatía (ponerse en el lugar del otro) \\
\hline & Compartir (habilidades, posesiones materiales, dinero, recursos, tiempo, etc \\
\hline \multirow{3}{*}{$\begin{array}{l}\text { Bien común y } \\
\text { equidad social }\end{array}$} & Igualdad (iguales oportunidades para todos) \\
\hline & $\begin{array}{l}\text { Acción social (eliminar y apoyar la eliminación de obstáculos al desarrollo } \\
\text { humano) }\end{array}$ \\
\hline & Distribución equitativa (todos tienen acceso igualitario a los recursos) \\
\hline \multirow{3}{*}{$\begin{array}{l}\text { Desarrollo } \\
\text { sostenible y medio } \\
\text { ambiente }\end{array}$} & Orden social (estabilidad en la sociedad) \\
\hline & Conciliación (política de estado basada en la resolución pacífica de conflictos) \\
\hline & $\begin{array}{l}\text { Protección del medio ambiente (intervención consciente y deliberada para } \\
\text { solucionar los problemas ambientales) }\end{array}$ \\
\hline \multirow{3}{*}{$\begin{array}{l}\text { Aceptación y } \\
\text { aprecio de la } \\
\text { diversidad }\end{array}$} & $\begin{array}{l}\text { Valoración de la diversidad (reconocer las diferencias como una oportunidad } \\
\text { de aprendizaje y crecimiento personal) }\end{array}$ \\
\hline & $\begin{array}{l}\text { Tolerancia de culto (respeto a las, creencias de los demás cuando son } \\
\text { diferentes o contrarias a las propias) }\end{array}$ \\
\hline & Tolerancia sexual (aceptación de la preferencia homo y heterosexuales) \\
\hline \multirow[t]{3}{*}{$\begin{array}{l}\text { Compromiso con la } \\
\text { verdad }\end{array}$} & $\begin{array}{l}\text { Pensamiento crítico (examen y juicio racional y continuo acerca de alguien } \\
\text { o algo) }\end{array}$ \\
\hline & Honestidad (apego a la verdad ) \\
\hline & $\begin{array}{l}\text { Honestidad social (poner de manifiesto algo oculto y por lo común } \\
\text { reprochable) }\end{array}$ \\
\hline \multirow[t]{3}{*}{ Integridad } & $\begin{array}{l}\text { Coherencia (guardar correspondencia lógica con los principios que se } \\
\text { profesa) }\end{array}$ \\
\hline & $\begin{array}{l}\text { Competencia (pericia, aptitud, idoneidad para hacer algo o intervenir en un } \\
\text { asunto determinado) }\end{array}$ \\
\hline & Rectitud (justo, severo e intachable en su conducta) \\
\hline \multirow[t]{3}{*}{ Excelencia } & $\begin{array}{l}\text { Perseverancia (mantenerse constante en la consecución de los objetivos } \\
\text { propuestos) }\end{array}$ \\
\hline & Autociplina (autocontrol, resistencia a las tentaciones) \\
\hline & Capacidad (competente, eficaz, eficiente) \\
\hline \multirow[t]{3}{*}{$\begin{array}{l}\text { Interdependencia e } \\
\text { interdisciplinaridad }\end{array}$} & $\begin{array}{l}\text { Integración (buscar una perspectiva que sintetice distintos tipos de } \\
\text { conocimientos) }\end{array}$ \\
\hline & Cooperación (coordinar acciones para alcanzar metas comunes) \\
\hline & Intercambio (disposición a entregar y recibir distinta información) \\
\hline
\end{tabular}




\section{Anexo B: Ejemplos de ítems Escala Actitudes CAVSR}

\begin{tabular}{|c|c|}
\hline \multicolumn{2}{|c|}{\begin{tabular}{|l|l|} 
Dimensiones valóricas Ejemplos de ítems \\
\end{tabular}} \\
\hline \multirow{3}{*}{$\begin{array}{l}\text { Ciudadanía, } \\
\text { democracia y } \\
\text { participación. }\end{array}$} & Creo que es necesario que nos involucremos en las actividades de nuestra comunidad \\
\hline & La posibilidad de estar inscrito en el registro electoral me llena de satisfacción \\
\hline & $\begin{array}{l}\text { Evito asistir a asambleas estudiantiles cuando tengo la posibilidad de hacer algo más } \\
\text { entretenido }\end{array}$ \\
\hline \multirow[t]{3}{*}{$\begin{array}{l}\text { Dignidad de la } \\
\text { persona }\end{array}$} & $\begin{array}{l}\text { Pienso que el crecimiento y desarrollo personal constituyen más bien una opción que } \\
\text { un derecho de las personas }\end{array}$ \\
\hline & $\begin{array}{l}\text { Me enorgullece reconocer que las personas tenemos distintos derechos de acuerdo con } \\
\text { los méritos que hemos hecho para alcanzarlos }\end{array}$ \\
\hline & Demuestro respeto a las personas, solo si ellas me parecen dignas de ello \\
\hline \multirow[t]{3}{*}{ Libertad } & $\begin{array}{l}\text { Creo que las personas deberían esforzarse por expresar menos lo que piensan para ser } \\
\text { aceptadas en cualquier grupo social }\end{array}$ \\
\hline & Me da miedo cuando se deja que ciertas personas decidan por si solas por su futuro \\
\hline & Suelo escoger cosas guiado por el consenso, postergando mis intereses \\
\hline \multirow{3}{*}{$\begin{array}{l}\text { Solidaridad y } \\
\text { sociabilidad para la } \\
\text { convivencia }\end{array}$} & $\begin{array}{l}\text { Creo que dedicar parte de mi tiempo para atender las necesidades de las personas es } \\
\text { un compromiso que es necesario asumir }\end{array}$ \\
\hline & Me gustaría formar parte de una organización de ayuda social \\
\hline & Colaboraría en la organización de alguna campaña solidaria \\
\hline \multirow{3}{*}{$\begin{array}{l}\text { Bien común y } \\
\text { equidad social }\end{array}$} & Pienso que todos tenemos derecho a las mismas cosas \\
\hline & $\begin{array}{l}\text { Valoro las acciones del gobierno u otras instituciones que van en beneficio de la } \\
\text { integración social }\end{array}$ \\
\hline & $\begin{array}{l}\text { Formaría un grupo de personas que velara por desarrollar potencialidades en las } \\
\text { personas con menos oportunidades }\end{array}$ \\
\hline \multirow{3}{*}{$\begin{array}{l}\text { Desarrollo } \\
\text { sostenible y medio } \\
\text { ambiente }\end{array}$} & Pienso que la protección del medio ambiente es solo una moda \\
\hline & Me alegra la idea de que existan políticas que regulen los temas ambientales. \\
\hline & Estaría dispuesto a separar la basura de mi casa para reciclarla. \\
\hline \multirow{3}{*}{$\begin{array}{l}\text { Aceptación y } \\
\text { aprecio de la } \\
\text { diversidad }\end{array}$} & Pienso que en algunos casos discriminar a otros puede ser justificable. \\
\hline & Siento que algunas religiones otorgan mejor guía que otras \\
\hline & Estaría dispuesto a aceptar a una persona homosexual en mi grupo de amigos \\
\hline \multirow{3}{*}{$\begin{array}{l}\text { Compromiso con la } \\
\text { verdad }\end{array}$} & Me da rabia que exista publicidad engañosa en los medios de comunicación \\
\hline & Siento que las mentiras pequeñas pueden salvar algunas situaciones poco agradables \\
\hline & $\begin{array}{l}\text { Estaría dispuesto a ocultar información poco relevante sobre mi desempeño si de ello } \\
\text { dependiera tener éxito en una tarea }\end{array}$ \\
\hline \multirow[t]{3}{*}{ Integridad } & Pienso que faltar a la ética en el ejercicio profesional para obtener éxito es reprochable \\
\hline & $\begin{array}{l}\text { Siento que ser transparente y honesto depende de la situación en que se encuentren } \\
\text { las personas }\end{array}$ \\
\hline & $\begin{array}{l}\text { Procuro mantener una coherencia entre mis principios y mis acciones en la vida } \\
\text { cotidiana }\end{array}$ \\
\hline \multirow[t]{3}{*}{ Excelencia } & Pienso que si me propongo algo debo hacerlo con calidad \\
\hline & Me molesta exigirme siempre al $100 \%$ en lo que hago \\
\hline & $\begin{array}{l}\text { Suelo hacer mayores esfuerzos en mi trabajo solo cuando sé que la evaluación está } \\
\text { cerca }\end{array}$ \\
\hline \multirow[t]{3}{*}{$\begin{array}{l}\text { Interdependencia e } \\
\text { interdisciplinaridad }\end{array}$} & $\begin{array}{l}\text { Creo que mi carrera me permite abordar las problemáticas sin necesidad de apoyo de } \\
\text { otras disciplinas }\end{array}$ \\
\hline & Me entretiene abordar una problemática desde distintas perspectivas de estudio \\
\hline & $\begin{array}{l}\text { Trabajar con profesionales de distintas áreas más que apoyar el trabajo, lo entorpece } \\
\text { por la complejidad que le confiere }\end{array}$ \\
\hline
\end{tabular}




\section{Anexo C: Dimensiones valóricas de la responsabilidad social (equipo Universidad Construye País, 2002)} \begin{tabular}{l} 
Dignidad de la persona: Considera a la persona como un ser que nace libre e igual en dignidad, derechos \\
y deberes, viviendo su pertenencia al género humano desde varias dimensiones: físico-biológica, psíquico- \\
espiritual y sociocultural. \\
\hline $\begin{array}{l}\text { Libertad: Es la potencia de vida de la persona humana por medio de la cual cada uno es capaz de autorrealizarse } \\
\text { en todas las dimensiones de su dignidad, haciendo concreta la responsabilidad sobre su propia vida y la de } \\
\text { la sociedad a la que pertenece. }\end{array}$ \\
\hline $\begin{array}{l}\text { Ciudadanía, democracia y participación: Práctica de la persona que se hace consciente de los derechos y } \\
\text { obligaciones que adquiere por el mero hecho de pertenecer a una comunidad social y/o política, de carácter } \\
\text { nacional, regional o local, y mediante la cual se contribuye a la construcción y cambio de la misma. }\end{array}$ \\
\hline $\begin{array}{l}\text { Sociabilidad y solidaridad para la convivencia: se refiere a la participación en la comunidad comprometiéndose } \\
\text { a responder a las obligaciones que supone la convivencia mutua y al mismo tiempo, asumir gratuitamente las } \\
\text { necesidades de los otros y, así, practicar la solidaridad como la dimensión estética y creadora de la convivencia. }\end{array}$ \\
\hline Bien común y equidad social: Conjunto de condiciones materiales, socioculturales y espirituales que permiten \\
a la sociedad y a quienes la integran acceder a una existencia digna y a una cada vez mejor calidad de vida. \\
Igualdad de oportunidades para satisfacer necesidades y desarrollar las capacidades de personas y grupos, \\
eliminando y apoyando la remoción de los obstáculos de carácter económico y social, así como los culturales \\
y políticos, que impiden el desarrollo humano.
\end{tabular}

Desarrollo sostenible y medio ambiente: Proceso que permite que las culturas humanas puedan desarrollarse, y que los efectos de la actividad humana (económica) se mantengan dentro de sus límites, de modo que no se destruya la diversidad, la complejidad y el funcionamiento del sistema ecológico que sirve de sostenimiento a la vida, así como al capital social que asegura la gobernabilidad democrática.

Aceptación y aprecio de la diversidad: Capacidad de valorar al otro e integrarlo a la sociedad sin discriminación por razones de raza, sexo, edad, condición religiosa, social ni política.

Compromiso con la verdad: Conjunto de principios éticos para su obtención: respeto a las verdades de las distintas dimensiones del conocimiento; la humildad; la honestidad para declarar los límites de la verdad alcanzada, y la prudencia, para no hacer de ella un mandato que pone en riesgo la dignidad humana.

Integridad: Cualidad de persona que es coherente con sus principios y valores, tanto en sus declaraciones como en sus acciones, siendo rectas, transparentes y honestas.

Excelencia: Expresión de calidad o bondad superior que distingue y hace objeto de especial aprecio al quehacer de personas u organizaciones. Atañe tanto a los fines, medios y procedimientos y se expresa en la gestión y en las funciones universitarias.

Interdependencia e interdisciplinariedad: Relación dinámica entre el individuo y la sociedad, que reconoce su mutua necesidad y que, por ello, requiere un diálogo permanente. 